\title{
Ivosidenib - Another Feather in the Hat of Treatment for Acute Myeloid Leukemia
}

\begin{abstract}
The treatment of acute myeloid leukemia has undergone a paradigm shift in the past few years. Multiple new targeted and nontargeted agents have been approved in the recent past. Isocitrate dehydrogenase (IDH) mutation is one such target that has been identified, and two new drugs, ivosidenib and enasidenib have been approved. The former is an IDH1 inhibitor, and the latter is an IDH2 inhibitor. The mechanism of action, key trials, adverse events, and monitoring of ivosidenib has been discussed in this article.
\end{abstract}

Keywords: Isocitrate dehydrogenase inhibitors, ivosidenib, new drugs in acute myeloid leukemia

\section{Introduction}

Acute myeloid leukemia (AML) is an aggressive hematologic malignancy. Until the recent past, the standard treatment of newly diagnosed AML in fit patients was induction (1-2 cycles) with cytarabine and daunorubicin followed by consolidation (2-3 cycles) with intermediate or high dose cytarabine. Allogeneic stem cell transplantation is done as consolidation in intermediate- and high-risk category patients. Despite all the above expensive treatments, ${ }^{[1]}$ the cure rates for AML were very modest. ${ }^{[2]}$ Moreover, in elderly patients who are not fit for such aggressive treatments, the treatment is aimed at just prolonging life and controlling symptoms with agents such as decitabine, azacytidine, and low-dose cytarabine..$^{[3]}$

Of late, there have been multiple new targeted drugs that have been approved for use in AML, especially in elderly patients. One such group of drugs is isocitrate dehydrogenase (IDH) inhibitors. ${ }^{[4]}$ There are two IDH inhibitors approved for use. Ivosidenib is an IDH1 inhibitor, and enasidenib is an IDH2 inhibitor. This is a review of ivosidenib.

\section{Mechanism of Action and Pharmacokinetics}

IDH1 gene is located on the $2 \mathrm{p} 33.3$ chromosome. It encodes for an enzyme

This is an open access journal, and articles are distributed under the terms of the Creative Commons Attribution-NonCommercial-ShareAlike 4.0 License, which allows others to remix, tweak, and build upon the work non-commercially, as long as appropriate credit is given and the new creations are licensed under the identical terms.

For reprints contact: WKHLRPMedknow_reprints@wolterskluwer.com called IDH1, which is located in the cytoplasm and peroxisomes. This enzyme converts isocitrate to alpha-ketoglutarate, which produces nicotinamide adenine dinucleotide phosphate (NADPH). NADPH is required for many cellular processes such as fat metabolism and scavenging of reactive oxygen species. Cytogenetically, normal AML patients harbor IDH1 mutations in about $6 \%-20 \%{ }^{[4-6]}$ The most common mutation in IDH1 gene is located in the arginine 132 (R132) residue. This mutation results in hypermethylation of DNA and histones, which in turn, causes an arrest in the differentiation of the myeloid series. Ivosidenib is an IDH1 inhibitor that inhibits the mutated IDH1 and causes a release of the differentiation arrest.

The metabolism of ivosidenib is by the CYP3A4 enzyme, and it is primarily excreted in feces, and the terminal half-life of ivosidenib is $93 \mathrm{~h}$. There is a high risk of developing QT prolongation and arrhythmias when ivosidenib is coadministered with other drugs, which cause QT prolongation and CYP3A4 enzyme inhibitors. Ivosidenib itself induces the CYP3A4 enzyme. This may lead to a decrease in the efficacy of the azole group of the antifungal drug, which is commonly coadministered in AML. Caution must be taken in patients using oral contraceptives along with ivosidenib, as there may be a decrease in efficacy due to the induction of the CYP3A4 enzyme.

How to cite this article: Perumal Kalaiyarasi $\mathrm{J}$ Ivosidenib - Another feather in the hat of treatment for acute myeloid leukemia. Indian J Med Paediatr Oncol 2020;41:552-4.

\section{Jayachandran Perumal Kalaiyarasi}

Department of Medical Oncology, Cancer Institute (WIA), Chennai, Tamil Nadu, India

Submitted: $26-\mathrm{Feb}-2020$ Revised: 01-May-2020 Accepted: 03-May-2020 Published: 29-Aug-2020

\section{Address for correspondence:} Dr. Jayachandran Perumal Kalaiyarasi,

Department of Medical Oncology, Cancer Institute (WIA), 38, Sardar

Patel Road, Guindy,

Chennai - 600 036, Tamil Nadu, India.

E-mail:dr.pkjayachandran@ gmail.com

Access this article online

Website: www.ijmpo.org

DOI: 10.4103/ijmpo.ijmpo_81_20 Quick Response Code:

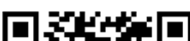

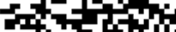
$1+2, y$

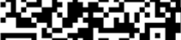
argirst 


\section{Key Trials}

\section{Study AG120-C-001 (NCT 02074839)}

This is an ongoing phase 1 single-arm, multicenter, dose escalation and expansion trial looking at safety, pharmacokinetics, pharmacodynamics, and clinical activity of single-agent oral ivosidenib (AG120) in advanced hematologic malignancies with IDH1 mutation. ${ }^{[7]}$ This trial included 179 relapsed/refractory AML patients. Complete response (CR) or complete response with partial hematologic recovery $(\mathrm{CRh})$ was seen in $30.4 \%{ }^{[8]}$ This trial also included 28 previously untreated patients with therapy-related AML and AML with myelodysplasia, of which 12 patients $(42.9 \%)$ achieved a CR or $\mathrm{CRh} \cdot{ }^{[9]}$ Two patients underwent allogeneic stem cell transplantation after CR with ivosidenib. Based on this trial, the Food and Drug Administration (FDA) has approved ivosidenib in newly diagnosed AML patients $\geq 75$ years of age with IDH1 mutation and relapsed or refractory AML patients with IDH1 mutation.

\section{Study AG120-C-009 (NCT 03173248)}

This is an ongoing phase 3 multicenter randomized double-blinded trial looking at ivosidenib with azacytidine versus placebo with azacytidine in untreated AML patients of $\geq 18$ years of age with IDH1 mutation. This trial has an estimated enrollment of 392 patients and expected to be completed by June 2022. ${ }^{[10]}$

\section{Maintenance therapy after allogeneic stem cell transplantation (NCT 03564821)}

This is an ongoing phase 1 study evaluating the use of ivosidenib as maintenance after allogeneic stem cell transplantation in AML, myelodysplastic syndromes, and chronic myelomonocytic leukemia patients with IDH1 mutation. It has estimated to enroll 22 patients. ${ }^{[11]}$

\section{Diagnostic Test}

The FDA has approved a companion diagnostic test for testing IDH1 mutation called Abbott Real Time TM IDH1 assay.

\section{Dosage}

Ivosidenib comes as a $250 \mathrm{mg}$ tablet. The recommended dosage is $500 \mathrm{mg}$ once daily until progression or unacceptable toxicity. The treatment should be continued for at least 6 months to show a clinical response.

\section{Adverse Events and Special Caution}

The common adverse events are diarrhea (53\%), fatigue (47\%), edema (26\%), anemia (26\%), leukocytosis (26\%), dyspnea (24\%), and hypomagnesemia $(24 \%) .{ }^{[9]}$

Differentiation syndrome (DS) is a unique adverse effect of this novel drug. It occurs in about $20 \%$ of the patients. DS is treated using standard drugs such as corticosteroids, diuretics, and hydroxyurea (in case of leukocytosis). DS seldom requires permanent discontinuation of the therapy. ${ }^{[12]}$

Caution must be taken if a patient develops QTc prolongation, as it may lead to dangerous arrhythmias. Dose reduction to $250 \mathrm{mg}$ once daily is recommended for patients who develop QTc $>500 \mathrm{~ms}$ after normalization.

Guillain-Barre syndrome is a very rare, but dangerous adverse effect of ivosidenib, which was reported in $<1 \%$ of the patients in the trial. It requires permanent discontinuation of the drug.

If a strong CYP3A4 inhibitor is coadministered, the dose must be reduced to $250 \mathrm{mg}$ once daily.

\section{Monitoring}

Blood counts and biochemical tests are to be done once weekly in the $1^{\text {st }}$ month, once in 2 weeks in the $2^{\text {nd }}$ month, and then once monthly. Creatinine phosphokinase should be monitored once weekly for the $1^{\text {st }}$ month. ECG should be monitored once weekly in the $1^{\text {st }}$ month and then once monthly. ${ }^{[13]}$

\section{Other Indications}

Ivosidenib has also been indicated off label in IDH1 mutant gliomas. Ivosidenib has also shown to have activity in IHD1 mutant cholangiocarcinoma and chondrosarcoma. ${ }^{[14]}$

\section{Conclusion}

Ivosidenib is a novel targeted drug that is a promising option for IDH1 mutant AML patients.

\section{Financial support and sponsorship}

Nil.

\section{Conflicts of interest}

There are no conflicts of interest.

\section{References}

1. Philip C, George B, Ganapule A, Korula A, Jain P, Alex AA, et al. Acute myeloid leukaemia: Challenges and real world data from India. Br J Haematol 2015; 170:110-7.

2. Kalaiyarasi JP, Ganesan P, Kannan K, Ganesan TS, Radhakrishnan V, Dhanushkodi M, et al. Outcomes of intensive treatment of adult acute myeloid leukemia patients: A retrospective study from a single centre. Indian J Hematol Blood Transfus 2019;35:248-54.

3. Pant M, Bhatt VR. Early mortality and survival in older adults with acute myeloid leukemia. Int J Hematol Oncol 2017;6:61-3.

4. Megías-Vericat JE, Ballesta-López O, Barragán E, Montesinos P. IDH1-mutated relapsed or refractory AML: Current challenges and future prospects. Blood Lymphat Cancer Targets Ther 2019;9:19-32.

5. Patel KP, Ravandi F, Ma D, Paladugu A, Barkoh BA, Medeiros LJ, et al. Acute myeloid leukemia with IDH1 or IDH2 mutations: Frequency and clinicopathologic features. Am J Clin Pathol 2011;135:35-45. 
6. DiNardo CD, Ravandi F, Agresta S, Konopleva M, Takahashi K, Kadia $\mathrm{T}$, et al. Characteristics, clinical outcome, and prognostic significance of IDH mutations in AML. Am J Hematol 2015;90:732-6.

7. Study of Orally Administered AG-120 in Subjects with Advanced Hematologic Malignancies with an IDH1 Mutation-Full Text View-ClinicalTrials. Available from: https://clinicaltrials.gov/ct2/ show/NCT02074839. [Last accessed on 2020 Feb 26].

8. DiNardo CD, Stein EM, Botton S de, Roboz GJ, Altman JK, Mims AS, et al. Durable remissions with ivosidenib in IDH1-mutated relapsed or refractory AML. N Engl J Med 2018;378:2386-98.

9. Prior DJ. A study of the electrophysiological properties of the incurrent siphonal valve muscle of the surf clam, Spisula solidissima. Comp Biochem Physiol A Comp Physiol 1975;52:607-10.

10. Study of AG-120 (Ivosidenib) vs. Placebo in Combination with Azacitidine in Patients with Previously Untreated Acute Myeloid Leukemia with an IDH1 Mutation-Full Text View-ClinicalTrials. Available from: https://clinicaltrials.gov/ct2/
show/NCT03173248. [Last accessed on 2020 Feb 26].

11. IDH1 Inhibition Using Ivosidenib as Maintenance Therapy for IDH1-Mutant Myeloid Neoplasms Following Allogeneic Stem Cell Transplantation-Full Text View-ClinicalTrials. Available from: https://clinicaltrials.gov/ct2/show/NCT03564821. [Last accessed on 2020 Feb 26].

12. Birendra K, DiNardo CD. Evidence for clinical differentiation and differentiation syndrome in patients with acute myeloid leukemia and IDH1 mutations treated with the targeted mutant IDH1 inhibitor, AG-120. Clin Lymphoma Myeloma Leuk 2016;16:460-5.

13. Highlights of Prescribing Information-Tibsovo ${ }^{\circledR}$ (Ivosidenib Tablets), for Oral Use; 2019. Available from: https://www. accessdata.fda.gov/drugsatfda_docs/label/2019/211192s001lbl. pdf.

14. Fan B, Mellinghoff IK, Wen PY, Lowery MA, Goyal L, Tap WD, et al. Clinical pharmacokinetics and pharmacodynamics of ivosidenib, an oral, targeted inhibitor of mutant IDH1, in patients with advanced solid tumors. Invest New Drugs 2020;38:433-44. 